OPEN ACCESS

Edited by:

Tânia Fernandes,

Universidade de Lisboa, Portugal

Reviewed by:

Rui Alexandre Alves,

University of Porto, Portugal Mariona Pascual,

University of Lisbon, Portugal

${ }^{*}$ Correspondence:

Lola Seyll

lola.seyl|@ulb.be

Specialty section:

This article was submitted to

Language Sciences,

a section of the journal

Frontiers in Psychology

Received: 16 June 2021

Accepted: 06 December 2021

Published: 16 February 2022

Citation:

Seyll L and Content A (2022)

Letter-Like Shape Recognition

in Preschool Children: Does

Graphomotor Knowledge Contribute?

Front. Psychol. 12:726454.

doi: 10.3389/fpsyg.2021.726454

\section{Letter-Like Shape Recognition in Preschool Children: Does Graphomotor Knowledge Contribute?}

\author{
Lola Seyll* and Alain Content \\ Laboratoire Cognition Langage et Développement, Centre de Recherche Cognition et Neurosciences, Université Libre de \\ Bruxelles (ULB), Brussels, Belgium
}

Based on evidence that learning new characters through handwriting leads to better recognition than learning through typing, some authors proposed that the graphic motor plans acquired through handwriting contribute to recognition. More recently two alternative explanations have been put forward. First, the advantage of handwriting could be due to the perceptual variability that it provides during learning. Second, a recent study suggests that detailed visual analysis might be the source of the advantage of handwriting over typing. Indeed, in that study, handwriting and composition -a method requiring a detailed visual analysis but no specific graphomotor activity- led to equivalent recognition accuracy, both higher than typing. The aim of the present study was to assess whether the contribution of detailed visual analysis is observed in preschool children and to test the variability hypothesis. To that purpose, three groups of preschool children learned new symbols either by handwriting, typing, or composition. After learning, children performed first a four-alternative recognition task and then a categorization task. The same pattern of results as the one observed in adults emerged in the four-alternative recognition task, confirming the importance of the detailed visual analysis in letter-like shape learning. In addition, results failed to reveal any difference across learning methods in the categorization task. The latter results provide no evidence for the variability hypothesis which would predict better categorization after handwriting than after typing or composition.

Keywords: letter representation, letter recognition, letter categorization, handwriting, graphic motor programs, visual analysis, perceptual variability

\section{INTRODUCTION}

New technologies are pervasive in our everyday life and computers are increasingly used at school (Wollscheid et al., 2016). The possibility of typewriting replacing handwriting from the very outset of literacy acquisition thus raises the question of its impact on reading development and on written language perception. Indeed, handwriting requires to reproduce a visual form by the execution of a sequence of fine movements that completely define the shape of the letter. This activity thus incurs very precise processing in terms of both visual and motor activity. By contrast, typewriting consists in a simple keypress based on the visual matching between two graphic forms. Interestingly, recent 
data suggest that simple visual exposure-even if massive-is not sufficient to yield a representation sufficiently detailed to lead to successful recognition. Wong et al. (2018) examined knowledge about the shape of the "looptail" $g$ allograph that is used in most print materials. They observed that skilled adult readers failed on simple tasks such as drawing the letter $g$ or identifying it among distractors. Even more surprisingly, despite extensive visual exposure to the looptail $g$ allograph, many skilled readers failed to simply recall its existence as an alternate form of the lowercase letter g. The authors suggested that the absence of writing experience with the looptail $g$ might be the source of its ensuing underspecified shape representation. These observations question the type of experience required to construct detailed and accurate shape representations of letters, with clear educational implications.

Indeed, letter recognition ability is an important predictor of subsequent reading skills (Näslund and Schneider, 1996; Scanlon and Vellutino, 1996; O'Connor and Jenkins, 1999; Lonigan et al., 2000; Foulin, 2005). Moreover, most current models of word recognition assume that letter recognition is an essential step in the flow of processing leading to word identification (McClelland and Rumelhart, 1981; Coltheart et al., 2001; Dehaene et al., 2005; Perry et al., 2007).

Longcamp and colleagues directly assessed the impact of typewriting on the construction of letter representation. They conducted behavioral studies that compared recognition performance observed after handwriting and after typewriting (Longcamp et al., 2005b, 2006, 2008). For both preschool children and adults, learning new characters through handwriting led to better recognition and orientation discrimination than learning through typewriting (see Seyll et al., 2020 for similar results). According to Longcamp et al. (2006), the advantage of handwriting is due to the contribution of the graphic motor programs-i.e., mental descriptions of the sequence of fine movements required to write the letter (van Galen, 1991; see Palmis et al., 2017, for a review)-constructed in memory through writing experience. More precisely, "the detection of a match or a mismatch between the perceived shape and the memorized motor program might contribute to the mirror-normal recognition processes and therefore explain the behavioral facilitation for the characters learned by handwriting" (Longcamp et al., 2006, p. 653). This process would be particularly helpful for letters that are ambiguous for the visual system such as mirror letters (e.g., b-d or p-q).

This interpretation thus assumes that joint reading and writing activities would gradually lead to a multimodal network of letter representation linking both the visual and the graphic motor codes (see for reviews Longcamp et al., 2010, 2016; James, 2017). In this embodied cognition perspective, one single sensory modality is sufficient to activate the entire distributed network which was engaged when the object was initially stored in memory (Wilson, 2002; Barsalou et al., 2003; Barsalou, 2008; Sullivan, 2018). The multimodal account of letter representation is supported by neuroimaging studies showing that the visual perception of letters activates precisely the same premotor area which is engaged during writing (Longcamp et al., 2003, 2005a; James and Gauthier, 2006). This premotor activation would reflect the automatic activation of the graphic motor program necessary to produce the perceived letter (Longcamp et al., 2003, 2005a).

Recently, however, several additional explanations of the advantage of handwriting over typing have been proposed. James and Engelhardt (2012; see also Li and James, 2016; James, 2017) proposed that handwriting would lead to broader and more abstract letter representations than other learning methods because it entails perceptual variability during learning. According to this hypothesis, facing varying exemplars would help identify the critical invariants and ignore irrelevant changes. The importance of perceptual variability during learning is not a new hypothesis. Indeed, according to several studies, comparison would play a critical role in the categorization of novel objects (e.g., Gentner and Namy, 1999; Namy and Gentner, 2002; Graham et al., 2010; Twomey et al., 2014). Some studies even suggested that the greater the variability among exemplars during learning, the better the generalization to new category instances (Posner and Keele, 1968; Perry et al., 2010). Handwriting is particular because in that case, the brain, the body, and the environment interact in a circular way ( $\mathrm{Li}$ and James, 2016). The efferent motor commands sent by the brain for producing a given letter lead to variable outputs-i.e., the handwritten productions-which in turn constitute variable environmental inputs for the visual system and reshape the letter category boundaries. In contrast to handwriting, typing does not provide such variability since it exposes the learner to one single and invariant prototypical exemplar of each character, at both output and input levels.

Indeed, a recent behavioral study shows that variability improves the learning of letters by revealing that 5-year-old children were better at letter categorization when they were exposed to multiple exemplars of the letters during learning than when they were exposed to one single exemplar, whatever the learning modality - free handwriting, tracing, or viewing ( $\mathrm{Li}$ and James, 2016). However, the advantage of perceptual variability arose whether the learning modality involved graphomotor activity or not. Moreover, it emerged whether the exemplars were self-produced, produced by another child, or simply typed. Hence, those results suggest that it is not the graphomotor activity per se that is the key factor explaining the facilitating effect but rather the perceptual variability that handwriting produces during learning.

A third interpretation has been proposed by Seyll et al. (2020). They suggested that the role of one component process, namely, the detailed visual analysis involved in handwriting, might have been underestimated in the advantage of handwriting over typing. More precisely, they assessed whether the detailed visual analysis required by handwriting but not by typing might contribute to the advantage of the former. To that purpose, they introduced a third learning method-called composition-requiring a detailed visual analysis but suppressing the graphomotor activity. During composition, participants received the set of elementary visual features used to construct the symbols and they were asked to reproduce the target symbol by selecting the appropriate features and assembling them together (as with a jigsaw puzzle). 
After learning, participants performed two recognition tests, a four-alternative forced-choice (4AFC) test and an old/new test. Distractors used in the $4 \mathrm{AFC}$ were visually close to the target symbol (e.g., mirror-reversed symbol and symbol with one feature mislocated). Distractors used in the old/new recognition test consisted exclusively in the mirror-images of the learned symbols (as in Longcamp et al., 2006, 2008). The results failed to reveal any clear advantage of handwriting over composition, both leading to better recognition than typing, thus suggesting a significant contribution of the detailed visual analysis in the advantage of handwriting over typing.

We recently discovered that a similar hypothesis had already been proposed and put to the test by Courrieu and De Falco (1989). ${ }^{1}$ They examined perceptual discrimination of Roman letters before and after learning. The learning conditions varied according to two orthogonally manipulated factors, analysis of target letters into segments, and dynamic tracing of the letters. In the analysis condition, which is very similar to our composition condition, preschool children received the target letter broken down into three segments on separate pieces of paper, and they had to recombine the pieces to reproduce the model. In the tracing condition, the children had to draw the target letter by following a trace on the worksheet. They improved significantly more from pre- to post-test when the learning involved analysis of letters into segments than when it did not, thus supporting the detailed visual analysis hypothesis, but the results failed to reveal any clear beneficial effect of tracing. However, tracing is known to be less effective than free copying (Naka, 1998). According to Naka (1998), the disadvantage of tracing over free copying might be due to the fact that, in contrast to handwriting, tracing does not require to generate and hold the image of the shape into memory. However, it is also likely that tracing does engage the graphomotor system to a lesser extent than free copying and does not lead to the storage of a graphomotor plan robust enough to facilitate discrimination. Nevertheless, given Naka's (1998) results, our choice of free copying provides a more appropriate test of the role of graphomotor knowledge.

The purpose of the present study was twofold. First, it aimed at further investigating the role of the detailed visual analysis inherent to handwriting by assessing whether the results observed with adults by Seyll et al. (2020) were generalizable to preschool children. To that purpose, children learned new symbols through handwriting, through typing, or through composition. After learning, they performed a 4AFC recognition task (as in Seyll et al., 2020). At the onset of reading acquisition, the visual system has to develop specific adaptations in order to effectively discriminate one letter from another. Indeed, learning to read impacts certain natural properties of the visual system. One such property is mirrorimage generalization, or mirror-invariance, the natural process of generalization across mirror images (Bornstein et al., 1978; Biederman and Cooper, 1991; Dehaene et al., 2010). In contrast to literate adults, prereaders have not yet overcome mirrorinvariance and often apply it to graphic shapes (Gibson et al., 1962; Cornell, 1985; Fernandes et al., 2016). As suggested by

\footnotetext{
${ }^{1}$ We thank the Editor for pointing us to that reference.
}

Longcamp et al., 2006, handwriting might be particularly helpful in overcoming mirror-invariance during literacy acquisition. If the graphic motor programs constructed through handwriting are the source of its advantage (graphomotor hypothesis), one might expect better recognition performance after handwriting than after typing and composition, both leading to equivalent recognition levels. In contrast, if the source of the handwriting advantage is the detailed visual analysis required by copying (visual analysis hypothesis), handwriting and composition should lead to equivalent recognition performance, both better than typing. Of course, the two hypotheses are not exclusive. If both graphic motor programs and detailed visual analysis contribute to the handwriting advantage (mixed hypothesis), handwriting should lead to more accurate recognition than composition, itself better than typing.

The absence of a clear advantage of handwriting over composition in Seyll et al. (2020) does not allow one to conclude that handwriting and composition would lead to equivalent performance in any recognition situation. Based on Li and James (2016) suggestion, it seemed plausible that despite equivalent recognition performance, handwriting would induce larger and richer representational categories as it is the only method that provides a diversity of exemplars during learning in the present experiment. It is worth noting that the $4 \mathrm{AFC}$ and Old/New tests may not be adequate to assess the richness of representations. The second purpose of the present study was to assess whether handwriting would improve categorization, as proposed by Li and James (2016). To this end, we also administered a categorization task like the one used by Li and James (2016). If perceptual variability improves the richness of letter representations, handwriting should affect categories and lead to better categorization performance than both other learning methods.

\section{MATERIALS AND METHODS}

\section{Participants}

Sixty-nine French-speaking kindergarteners (5 years 3 months to 6 years 3 months) took part in the experiment. There were 35 girls and 34 boys, and four left-handed participants. All were attending kindergarten in three different schools and had no known neurological diseases or psychological disorders. Participants were randomly assigned to one of the three groups. One group learned the symbols by handwriting, the second group by typing, and the third group by composition. Data from eight participants were discarded because they did not complete all the tests. There were 20 remaining participants in the handwriting group (mean age $=68.1$ months; $S D=3.68$ ), 22 in the typing group (mean age $=68.5$ months; $S D=2.92$ ), and 19 in the composition group (mean age $=69.5$ months; $S D=3.61$ ). Written informed consent was provided by the parents. The study was approved by the local ethics committee.

\section{Stimuli}

The method description is largely similar to Seyll et al. (2020) as the method is almost identical. Stimuli were symbols created 
from a set of six elementary features (Figure 1A). All possible symbols combining three features were generated, and we choose eight symbols in this library (Figure 1B). Stimuli were simpler than those used in Seyll et al. (2020). First, symbols were simplified in terms of the number of features. Indeed, whereas symbols used in Seyll et al. (2020) were composed of three, four, or five features, those used in the present study were all composed of three features. Then, only two elementary curves were used instead of four. Symbols used in the present study are referred to as "letter-like shapes" because they share the main characteristics with letters. They are the result of a combination of graphic elementary features, they can be handwritten, and the elementary features can be isolated.

\section{Procedure}

Three sessions were held 1 week apart. The learning phase was distributed over the first two sessions. The 4AFC task was administered immediately after the second learning session and again at the beginning of the third session. The categorization task was administered during the third session immediately after the 4AFC task. Before the first learning session, participants performed visuo-spatial and graphomotor tests-i.e., the visual perception and the motor coordination subtests of the VMI, respectively (Beery and Beery, 2004). All sessions took place in a quiet room at school and participants were tested individually.

In order to promote engagement, the tasks were embedded in a treasure hunt context. A little boy was displayed on the tablet screen and children were invited to help him to find a treasure. They were then explained that this little boy is living in a very distant country and that to help him, they would have to learn the letters used in his country. Moreover, to introduce the three sessions, children were shown a three-part totem and explained that each session would be rewarded by a part of it. Once the totem was completed, they could reach the treasure.

Stimulus presentation and response recording were programmed in Python using PsychoPy libraries. Stimuli were displayed on a Wacom Cintiq 13HDT tablet.

\section{Learning Phase}

All participants were asked to memorize eight unfamiliar symbols. In each learning session, there were three blocks each involving one random presentation of the eight symbols. Participants could take a break between blocks if needed. Before training, they received three practice trials with simple geometric shapes (a semicircle, a square, and a triangle). Feedback was given after each practice trial but not during learning.

The target symbol was horizontally centered on the tablet screen (as can be seen in Figure 2, its vertical position varied as a function of the learning method) and was displayed in black in a white $37-\mathrm{mm}$-wide area against a gray background. It stayed visible on the screen during the whole trial and the transition to the next trial was triggered by the participant. No constraint was imposed on production speed.

\section{Handwriting Method}

The target was centrally displayed during the entire trial (Figure 2A). At the start of each trial, participants were given a $100 \times 100 \mathrm{~mm}$ sheet, and had to copy one symbol per sheet within a square of $35 \times 35 \mathrm{~mm}$. Once the copy was done, the experimenter took the sheet back and hid it from view. No constraint was imposed on stroke direction or order. To trigger the next trial, participants clicked on the "next" button displayed in the lower-right corner of the screen. Response times from target onset until the "next" button press were recorded.

\section{Typing Method}

The screen was divided into three portions: the target symbol was displayed in the upper portion, the virtual keyboard in the middle portion, and the response area in the lower portion (Figure 2B). The virtual keyboard was composed of eight 17-mm-wide keys, corresponding to the eight target symbols. The position of the keys varied randomly across trials in order to promote an active visual research. The response area was of the same size and color as the target area. Those three portions were displayed during the entire trial. Participants had to find the key corresponding to the target symbol and click with the stylus on it. Responses triggered the apparition of the selected symbol in the response area for $1 \mathrm{~s}$ before the start of the next trial. Accuracy and response times from target onset until the key press were recorded. It should be noted that the typing method used in the present experiment is different from a typical typing task given that the position of the keys varied randomly across trials. In what follows, however, we will refer to it as "typing method" for the sake of clarity.

\section{Composition Method}

The screen was divided into three portions: the target symbol was displayed in the upper portion, the set of individual features in the middle portion, and the response area in the lower portion (Figure 2C). The middle section was composed of six features displayed in 20-mm-wide squares. The position of the features was kept constant across trials and across participants. The response area was of the same size and color as the target area. Those three portions were displayed during the entire trial. Participants had to compose the target symbol by selecting features in the features area and dragging them in the appropriate position in the response area. No constraint was imposed on stroke order. To trigger the next trial, participants clicked on the "next" button displayed in the lower-right corner of the screen. Productions and response times from target onset until "next" button press were recorded.

\section{Recognition Task}

Participants performed the 4AFC recognition task immediately after training (Immediate Test) and again 6-8 days later (Delayed Test). Each trial consisted of the presentation of four symbols: the learned symbol plus three distractors, i.e., the mirror image of the symbol (mirror symbol), the learned symbol with a feature displaced (transformed symbol), and the mirror image of the transformed symbol (mirror transformed symbol) (Figure 3). The four symbols were randomly displayed upper left, upper right, lower left, and lower right. The learned symbol could not occur more than twice in a row at the same position. Participants had to select the learned symbol by clicking on it with the stylus. 
A

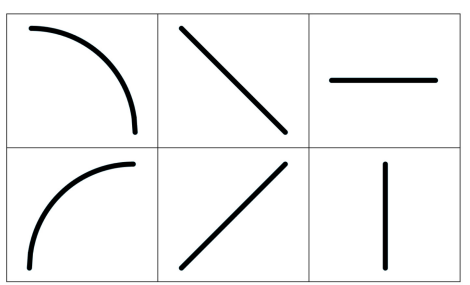

B

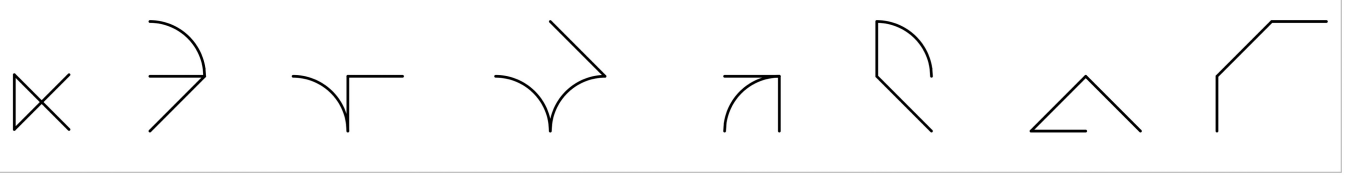

FIGURE 1 | (A) The six elementary features used to construct the symbol library. (B) The eight symbols to be learned.

A

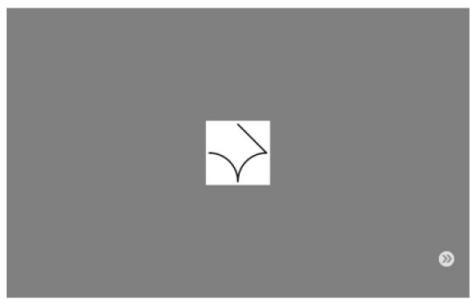

B

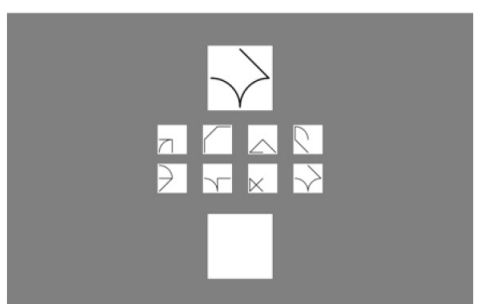

C

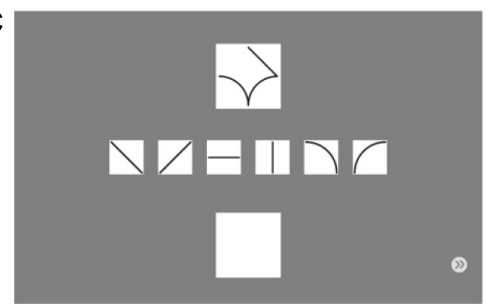

FIGURE 2 | One example of trial display for each learning method: (A) handwriting, (B) typing, and (C) composition.

LEARNED SYMBOL

FIGURE 3 | Examples of trials in the 4AFC recognition task.

As for the learning phase, symbols were displayed in black in a white $37-\mathrm{mm}$-wide area against a gray background. There were two blocks each involving the random presentation of the eight symbols and their distractors. Participants could take a break between blocks.

Each trial started with a centered fixation cross for $300 \mathrm{~ms}$, followed by a $200 \mathrm{~ms}$ gray screen. Then the four choices were displayed until the response. The intertrial interval was $500 \mathrm{~ms}$. The main dependent measure was accuracy. Response speed was not emphasized, although response times from target onset were also recorded.

\section{Categorization Task}

During the categorization task, children were required to sort 32 handwritten exemplars into categories corresponding to four of the learned symbols. Eight exemplars of each symbol were used. They were handwritten productions created by children of the same age range in a previous study (Figure 4). The exemplars 


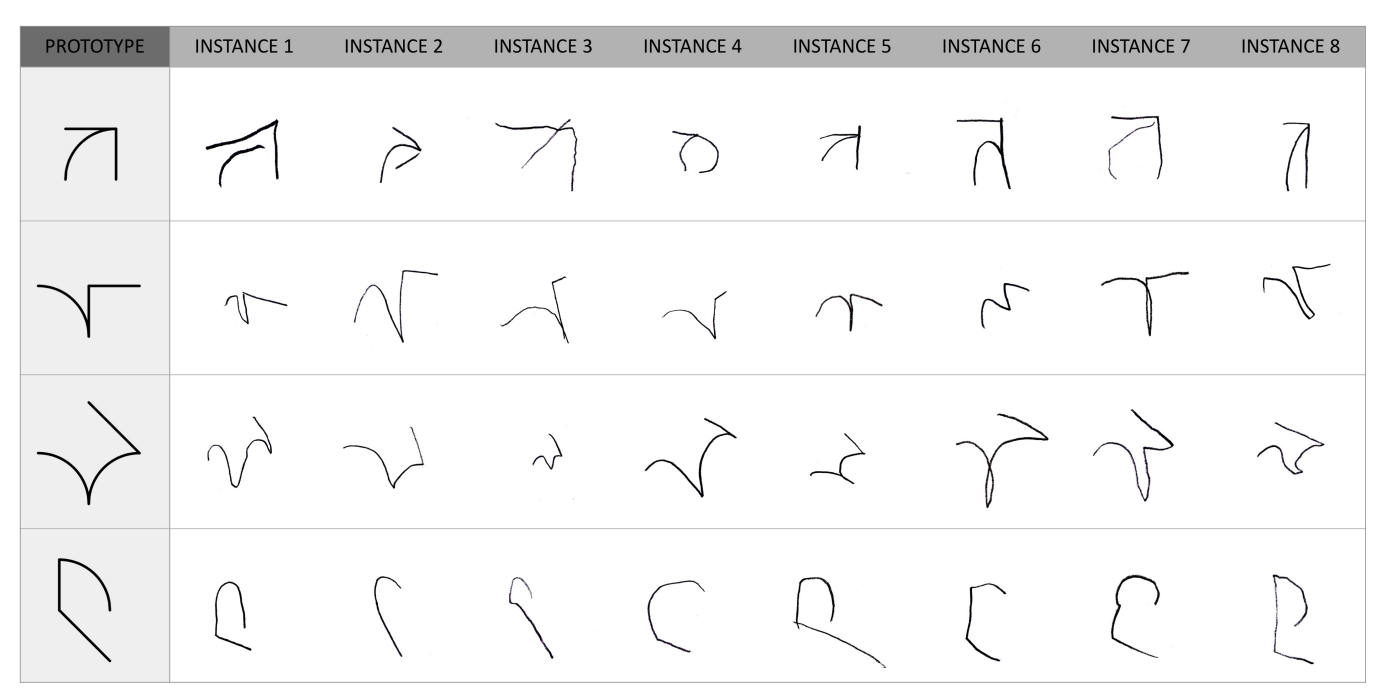

FIGURE 4 | The 32 handwritten productions used as experimental stimuli in the categorization task.

were displayed in black against a white background. Categories were instantiated by five house pictures displayed next to each other on the top of the screen. Four houses were assigned to the learned symbols, and one house was dedicated to the unlearned, new symbols. Across participants, the position of the four symbol-houses was randomized, but the "new symbols" house was fixed to the right. For a given participant, the position of the five houses remained constant. There were four blocks, each involving the random presentation of eight stimuli (two instances of each symbol). Participants could take a break between blocks.

Each trial started with the presentation of a new handwritten instance centered in the lower part of the screen. The five categories and the symbol instance remained visible during the entire trial (see Figure 5 for one example of trial). To select the category corresponding to the handwritten symbol, children had to click with the stylus on the corresponding house. This action triggered the instance's move to the selected house. The trial finished by the instance's entrance in the selected house and a short blast. If the child did not identify the symbol as belonging to any of the four symbol categories, he could select the "new symbols" category. The main dependent measure was accuracy. Response speed was not emphasized, although response times were also recorded.

Before the test, participants performed 10 practice trials with four simple geometric shapes categories (a circle, a heart, a square, and a triangle). Oral feedback was given after each practice trial but not during the test.

\section{RESULTS}

All data files are available at https://osf.io/a2893/. In frequentist analyses, handwriting was systematically contrasted to typing on the one hand and to composition on the other hand (as in Seyll et al., 2020). For both tasks, accuracy was analyzed in terms of proportion of correct responses. As no emphasis was put on response times, they were not further analyzed. Response times on correct trials were around 5,100 ms for both tasks, and they were similar for all learning methods.

\section{AFC Recognition Task}

Shapiro-Wilk normality tests on percentages of correct responses indicated no significant deviation from normality. Correct response rates (see Table 1) were submitted to an ANOVA with learning method (handwriting, composition, typing) as a between-subject factor and time of test as a within-subject factor (immediate, delayed). Mean percentages of correct responses are plotted in Figure 6A. The main effect of learning method was significant, $F(2,58)=15.006, p<0.001, \eta_{p}{ }^{2}=0.341$. A priori contrasts revealed a significantly higher proportion of correct responses after handwriting $(M=76.1 \%, S D=13.5)$ than after typing $(M=60.0 \%, S D=9.03), t=4.525, p<0.001$, but no significant difference between proportion of correct responses

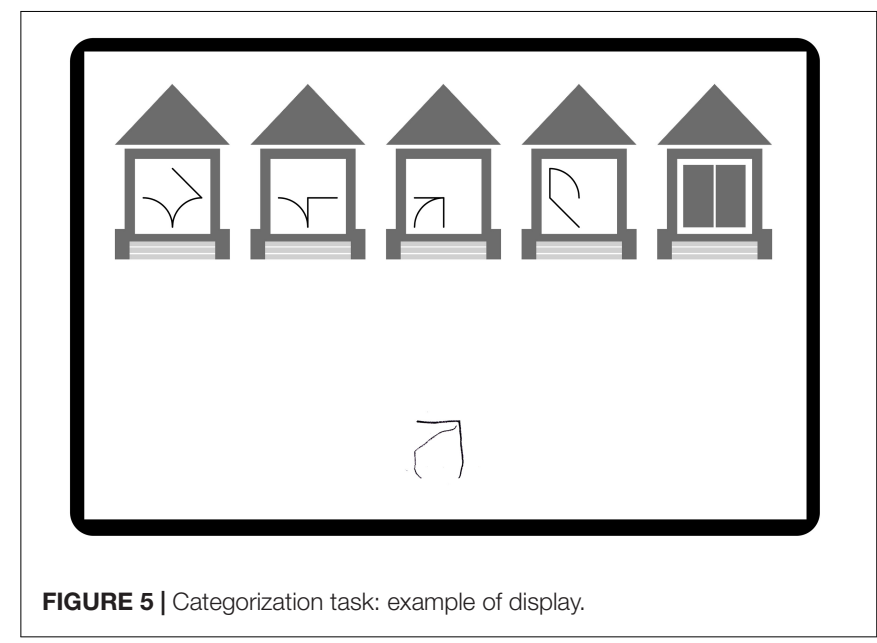


TABLE 1 | Performance for both recognition tasks across learning methods.

\section{Composition Handwriting Typing}

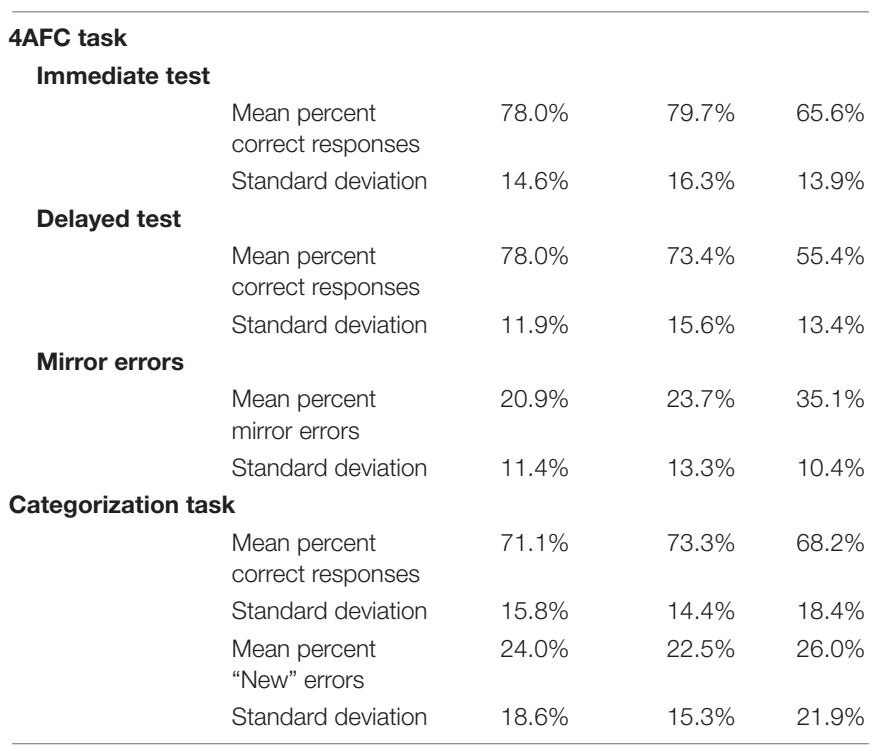

following handwriting and composition $(M=78.0 \%, S D=11.5)$, $t=0.380, p=0.705$. The main effect of time of test was significant, $F(1,58)=6.200, p=0.016, \eta_{p}{ }^{2}=0.097$, reflecting a higher rate of correct responses immediately after learning $(M=74.1 \%, S D=16.0)$ than 1 week later $(M=68.3 \%, S D=16.8)$. The interaction was not significant, $F(2,58)=1.816, p=0.17$, $\eta_{p}{ }^{2}=0.059$. To assess evidence in favor of an absence of difference between the handwriting and composition conditions, we additionally ran a Bayesian repeated-measures ANOVA, which produced concordant indications. Overall, the best model included time of test and learning method $\left(\mathrm{BF}_{10} \sim 10,000\right)$. Post hoc comparisons provided decisive evidence of differences between typing and both other conditions (Kass and Raftery, 1995, respectively, $\mathrm{BF}_{10} \sim 50,000$ for composition and $\mathrm{BF}_{10}$ $\sim 2,500$ for handwriting) and substantial evidence in favor of an absence of difference between composition and handwriting $\left(\mathrm{BF}_{10} \sim 0.254\right)$.

Error types are plotted in Figure 6B. On average, participants selected the mirror-image of the learned symbol on $26.9 \%$ of trials, the transformed symbol on $1.1 \%$ of trials, and the mirror transformed symbol on $1.0 \%$ of trials. A Shapiro-Wilk test on the rate of mirror-image choices indicated no significant deviation from normality. An ANOVA performed on the rate of mirror-image choices revealed a significant difference between learning methods, $F(2,58)=8.561, p<0.001, \eta_{p}{ }^{2}=0.228$. A significantly higher proportion of mirror-symbol choices was observed after typing $(M=35.1 \%, S D=10.4)$ than after handwriting $(M=23.7 \%, S D=13.3), t=3.144, p<0.001$, but there was no significant difference between handwriting and composition $(M=20.9 \%, S D=11.4), t=0.734, p=0.466$. The Bayesian ANOVA provided strong evidence in favor of an effect of learning method, $\mathrm{BF}_{10} \sim 58$. Post hoc tests again indicated differences between typing and both other conditions $\left(\mathrm{BF}_{10} \sim\right.$ 11 and 136, respectively, for handwriting and composition), and weak evidence in favor of the absence of difference between composition and handwriting $\left(\mathrm{BF}_{10} \sim 0.377\right)$.

\section{Categorization Task}

Shapiro-Wilk normality tests on percentages of correct responses indicated significant deviations from normality in two of the three groups. Hence, scores were submitted to a non-parametric ANOVA with learning method (handwriting, composition, and typing) as a between-subject factor. The Kruskal-Wallis test was non-significant $[H(2)=0.863, p=0.65]$. A Bayesian ANOVA similarly provided substantial evidence in favor of the null $\left(\mathrm{BF}_{10}\right.$ $\sim 0.195$ ). Mean percentages of correct responses and error rates are reported in Table 1.

On average, participants selected an erroneous category on $5.0 \%$ of trials and the "new symbols" category on $24.2 \%$ of trials. Shapiro-Wilk normality tests on rates "new symbols" choices indicated significant deviations from normality in two of the three groups. A non-parametric ANOVA performed on the rate of "new symbols" choices revealed no significant difference between learning methods, $H(2)=0.226, p=0.89$. The Bayesian ANOVA produced substantial evidence in favor of the null $\left(\mathrm{BF}_{10} \sim 0.15\right)$.

\section{DISCUSSION}

The changing habits introduced by the increasing use of digital devices in everyday life and at school raises the question of their impact on literacy acquisition. Indeed, one might wonder whether the reduced usage of handwriting at the very outset of reading acquisition has an impact on letter recognition, an essential step in word identification (McClelland and Rumelhart, 1981; Coltheart et al., 2001; Dehaene et al., 2005; Perry et al., 2007) generally considered as predictive of subsequent reading skills (Näslund and Schneider, 1996; Scanlon and Vellutino, 1996; O'Connor and Jenkins, 1999; Lonigan et al., 2000; Foulin, 2005).

A negative impact of keyboard use during letter learning has already been demonstrated. Indeed, multiple behavioral studies showed that handwriting is a more effective learning method and leads to better recognition and mirror discrimination than typing (Longcamp et al., 2005b, 2006, 2008; Seyll et al., 2020). Several interpretations of this finding have been proposed. First, the motor knowledge acquired through handwriting could contribute to recognition. Second, the perceptual variability entailed by handwriting could be the source of its advantage (James, 2017). Third, Seyll et al. (2020) argued that the detailed visual analysis required by handwriting might be a significant factor accounting for the advantage of handwriting over typing.

The first aim of the present study was to assess whether the results observed with adults by Seyll et al. (2020) were generalizable to preschool children. To that purpose, children learned new graphic shapes through handwriting, through typing, or through composition, and performed a $4 \mathrm{AFC}$ recognition task after learning. The second aim was to assess whether handwriting would lead to better categorization than typing and composition. To that purpose, we added a 
A

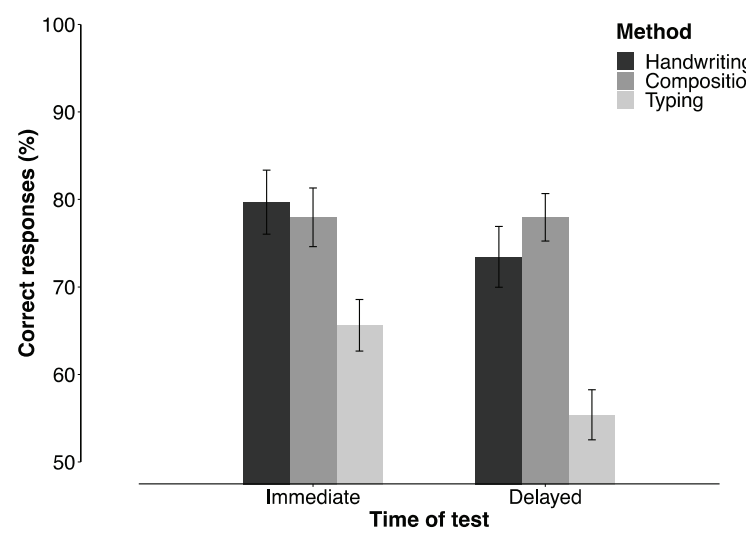

B

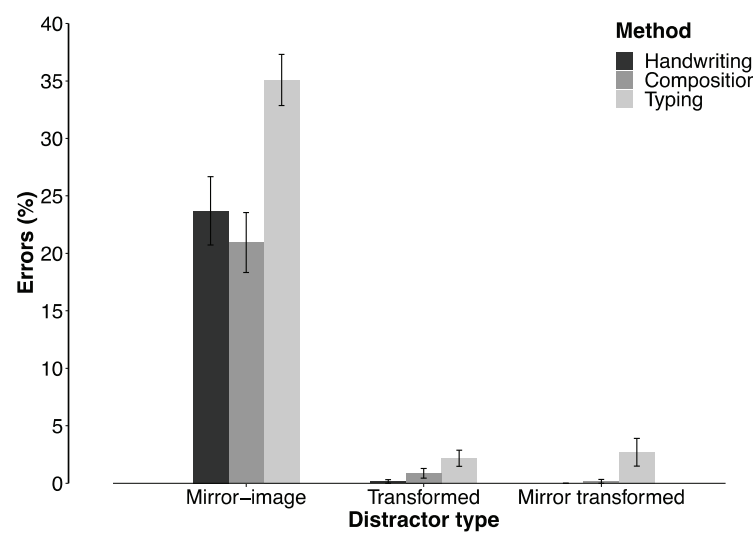

FIGURE 6 | (A) Mean percentage of correct responses for the immediate and delayed 4AFC test across learning methods. (B) Errors produced across the three learning methods. Error bars depict standard errors.

categorization task like the one used by Li and James (2016) at the end of the last session.

Recognition performance in the 4AFC task confirmed and corroborated the results observed in adults (Seyll et al., 2020), that is, higher recognition rates after handwriting and composition than after typing, with the two former leading to equivalent performance. Such a pattern is consistent with the idea that the detailed visual analysis plays an important role in letter-like shape learning and provides no evidence that the graphic motor programs, as such, contribute to letter recognition. Moreover, the present findings are in line with most current models of word recognition, which assume that letter recognition is a visual process based on elementary features extraction (McClelland and Rumelhart, 1981; Coltheart et al., 2001; Perry et al., 2007; Grainger et al., 2008). Indeed, the composition learning method used in the present study can be linked to the latter models because it precisely involves a visual focus on elementary features during learning.

Regarding the second issue, correct categorization rates failed to reveal any significant difference across the three learning methods. Moreover, if handwriting leads to richer and larger categories, new test exemplars should be less frequently rejected and a lower proportion of "new symbols" choices should be observed after handwriting than after typing and composition. However, as for the main analyses, the percentage of "new symbols" choices failed to reveal any significant difference across learning methods. Our results thus provide no evidence to confirm the variability hypothesis.

Mirror discrimination is essential for efficient reading. The present findings replicate the detrimental impact of typing on mirror-normal discrimination observed in previous studies (Longcamp et al., 2005b, 2006, 2008; Seyll et al., 2020). Mirrorimage errors are common at the onset of reading acquisition and dramatically decrease in the course of reading acquisition, between 5 and 7 years of age (Gibson et al., 1962; Nelson and Peoples, 1975; Fernandes et al., 2016). Several studies suggest that reversal errors are more frequent in children with developmental dyslexia during the first years of schooling (Wechsler and Hagin, 1964; Liberman et al., 1971; Wolff and Melngailis, 1996), and a recent study suggest that dyslexic children do not automatize mirror discrimination (Fernandes and Leite, 2017). There is thus cause for concern about a possible exacerbation of this weakness with the introduction of keyboarding at school. Dyslexic children might be more impacted by learning through typing than typically developing children and the predominant use of typing at school might constitute an additional risk factor for them.

In sum, the present findings clearly confirm that the detailed visual analysis is important in letter-like shape learning. It would yield detailed, accurate, and distinctive representations which support easy discrimination and identification. Under such a view, the association between letter perception and motor activation should be interpreted as a consequence of the learning experience and not as a necessary condition for encoding and recognition. Neither the present nor our previous studies (Seyll et al., 2020, 2021) showed an advantage for handwriting over composition and the Bayesian inference tests supported the null hypothesis. Even if it is too early to completely discard a possible contribution of graphic motor programs to letter recognition, our findings challenge the supporters of the graphomotor hypothesis to provide further evidence, over and above the influence of detailed analysis.

Regarding the potential implications of our conclusions for educational issues, it should be noted that the present learning situation differs in several ways from the usual school settings: the learning task was strictly visual and did not involve associations between graphic shapes and letter names or sounds, and the artificial symbols used here differ from real letters. Further studies using more ecological conditions and stimuli would be relevant to confirm the present findings.

While the present results provide no evidence in favor of a contribution of graphic motor programs and handwriting per se, it should, however, be noted that in the classroom, handwriting training and copy exercises may constitute the most natural way to promote such detailed visual analysis for most kids. Yet, 
handwriting might not constitute a suitable learning method for children with poor fine motor skills. Indeed, several studies revealed that poor fine motor skills are associated with poor reading skills (e.g., Grissmer et al., 2010; Cameron et al., 2012; Suggate et al., 2018, 2019). With normal adults, Seyll and Content (2020) showed that disturbing the graphomotor activity during symbol learning affects subsequent recognition and mirrornormal discrimination. The advantage of composition over typing observed in the present study might be exploited with children suffering from severe fine motor skills deficits. Indeed, it is plausible that children with poor fine motor skills would benefit from composition learning, as did the children of the present study. Further studies would be necessary to determine whether this is indeed the case.

\section{DATA AVAILABILITY STATEMENT}

All data files are available at https://osf.io/a2893/.

\section{REFERENCES}

Barsalou, L. W. (2008). Grounded cognition. Annu. Rev. Psychol. 59, 617-645. doi: 10.1146/annurev.psych.59.103006.093639

Barsalou, L. W., Kyle Simmons, W., Barbey, A. K., and Wilson, C. D. (2003). Grounding conceptual knowledge in modality-specific systems. Trends Cogn. Sci. 7, 84-91. doi: 10.1016/S1364-6613(02)00029-3

Beery, K. E., and Beery, N. A. (2004). The Beery-Buktenica Developmental Test of Visual-Motor Integration (Beery VMI): Administration, Scoring, and Teaching Manual, 5th Edn. Minneapolis, MN: NCS Pearson.

Biederman, I., and Cooper, E. E. (1991). Evidence for complete translational and reflectional invariance in visual object priming. Perception 20, 585-593. doi: $10.1068 / \mathrm{p} 200585$

Bornstein, M. H., Gross, C. G., and Wolf, J. Z. (1978). Perceptual similarity of mirror images in infancy. Cognition 6, 89-116. doi: 10.1016/0010-0277(78) 90017-3

Cameron, C. E., Brock, L. L., Murrah, W. M., Bell, L. H., Worzalla, S. L., Grissmer, D., et al. (2012). Fine motor skills and executive function both contribute to kindergarten achievement: fine motor and kindergarten achievement. Child Dev. 83, 1229-1244. doi: 10.1111/j.1467-8624.2012.01768.x

Coltheart, M., Rastle, K., Perry, C., Langdon, R., and Ziegler, J. (2001). DRC: a dual route cascaded model of visual word recognition and reading aloud. Psychol. Rev. 108, 204-256. doi: 10.1037//0033-295x.108.1.204

Cornell, J. M. (1985). Spontaneous mirror-writing in children. Can. J. Psychol. 39, 174-179. doi: $10.1037 / \mathrm{h} 0080122$

Courrieu, P., and De Falco, S. (1989). Segmental vs. dynamic analysis of letter shape by preschool children. Cahiers Psychol. Cogn. 9, 189-198.

Dehaene, S., Cohen, L., Sigman, M., and Vinckier, F. (2005). The neural code for written words: a proposal. Trends Cogn. Sci. 9, 335-341. doi: 10.1016/j.tics.2005. 05.004

Dehaene, S., Nakamura, K., Jobert, A., Kuroki, C., Ogawa, S., and Cohen, L. (2010). Why do children make mirror errors in reading? Neural correlates of mirror invariance in the visual word form area. NeuroImage 49, 1837-1848. doi: 10.1016/j.neuroimage.2009.09.024

Fernandes, T., and Leite, I. (2017). Mirrors are hard to break: a critical review and behavioral evidence on mirror-image processing in developmental dyslexia. J. Exp. Child Psychol. 159, 66-82.

Fernandes, T., Leite, I., and Kolinsky, R. (2016). Into the looking glass: literacy acquisition and mirror invariance in preschool and first-grade children. Child Dev. 87, 2008-2025. doi: $10.1111 /$ cdev.12550

Foulin, J. N. (2005). Why is letter-name knowledge such a good predictor of learning to read? Read. Writ. 18, 129-155. doi: 10.1007/s11145-004-5892-2

\section{ETHICS STATEMENT}

The studies involving human participants were reviewed and approved by Comité d'Éthique de la Faculté des Sciences Psychologiques et de l'Éducation, ULB. Written informed consent to participate in this study was provided by the participants' legal guardian/next of kin.

\section{AUTHOR CONTRIBUTIONS}

Both authors designed the experiment, analyzed the data and wrote the manuscript.

\section{ACKNOWLEDGMENTS}

The first author was a research fellow with the Belgian FRS-FNRS. The present article has been published with support from the Fondation Universitaire de Belgique.

Gentner, D., and Namy, L. L. (1999). Comparison in the development of categories. Cogn. Dev. 14, 487-513. doi: 10.1016/S0885-2014(99)00016-7

Gibson, E. J., Pick, A. D., Osser, H., and Gibson, J. J. (1962). A developmental study of discrimination of letter-like forms. J. Comp. Physiol. Psychol. 55, 897-906. doi: 10.1037/h0043190

Graham, S. A., Namy, L. L., Gentner, D., and Meagher, K. (2010). The role of comparison in preschoolers' novel object categorization. J. Exp. Child Psychol. 107, 280-290. doi: 10.1016/j.jecp.2010.04.017

Grainger, J., Rey, A., and Dufau, S. (2008). Letter perception: from pixels to pandemonium. Trends Cogn. Sci. 12, 381-387. doi: 10.1016/j.tics.2008.06.006

Grissmer, D., Grimm, K. J., Aiyer, S. M., Murrah, W. M., and Steele, J. S. (2010). Fine motor skills and early comprehension of the world: two new school readiness indicators. Dev. Psychol. 46, 1008-1017. doi: 10.1037/a0020104

James, K. H. (2017). The importance of handwriting experience on the development of the literate brain. Curr. Direct. Psychol. Sci. 26, 502-508. doi: $10.1177 / 0963721417709821$

James, K. H., and Engelhardt, L. (2012). The effects of handwriting experience on functional brain development in pre-literate children. Trends Neurosci. Educ. 1, 32-42. doi: 10.1016/j.tine.2012.08.001

James, K. H., and Gauthier, I. (2006). Letter processing automatically recruits a sensory-motor brain network. Neuropsychologia 44, 2937-2949. doi: 10.1016/j. neuropsychologia.2006.06.026

Kass, R. E., and Raftery, A. E. (1995). Bayes factors. J. Am. Stat. Assoc. 90, 773-795. doi: 10.1080/01621459.1995.10476572

Li, J. X., and James, K. H. (2016). Handwriting generates variable visual output to facilitate symbol learning. J. Exp. Psychol. Gen. 145, 298-313. doi: 10.1037/ xge0000134

Liberman, I. Y., Shankweiler, D., Orlando, C., Harris, K. S., and Berti, F. B. (1971). Letter confusions and reversals of sequence in the beginning reader: implications for Orton's theory of developmental dyslexia. Cortex 7, 127-142. doi: 10.1016/s0010-9452(71)80009-6

Longcamp, M., Anton, J.-L., Roth, M., and Velay, J.-L. (2003). Visual presentation of single letters activates a premotor area involved in writing. NeuroImage 19, 1492-1500. doi: 10.1016/S1053-8119(03)00088-0

Longcamp, M., Anton, J.-L., Roth, M., and Velay, J.-L. (2005a). Premotor activations in response to visually presented single letters depend on the hand used to write: a study on left-handers. Neuropsychologia 43, 1801-1809. doi: 10.1016/j.neuropsychologia.2005.01.020

Longcamp, M., Boucard, C., Gilhodes, J.-C., and Velay, J.-L. (2006). Remembering the orientation of newly learned characters depends on the associated writing knowledge: a comparison between handwriting and typing. Hum. Mov. Sci. 25, 646-656. doi: 10.1016/j.humov.2006.07.007 
Longcamp, M., Boucard, C., Gilhodes, J.-C., Anton, J.-L., Roth, M., Nazarian, B., et al. (2008). Learning through hand- or typewriting influences visual recognition of new graphic shapes: behavioral and functional imaging evidence. J. Cogn. Neurosci. 20, 802-815. doi: 10.1162/jocn.2008.20504

Longcamp, M., Lagarrigue, A., and Velay, J.-L. (2010). Contribution de la motricité graphique à la reconnaissance visuelle des lettres. Psychol. Franç. 55, 181-194. doi: 10.1016/j.psfr.2010.03.001

Longcamp, M., Velay, J., Berninger, V. W., and Richards, T. (2016). Neuroanatomy of handwriting and related reading and writing skills in adults and children with and without learning disabilities: French-American Connections. Pratiques 171-172:3175. doi: 10.4000/pratiques.3175

Longcamp, M., Zerbato-Poudou, M.-T., and Velay, J.-L. (2005b). The influence of writing practice on letter recognition in preschool children: a comparison between handwriting and typing. Acta Psychol. 119, 67-79. doi: 10.1016/j. actpsy.2004.10.019

Lonigan, C. J., Burgess, S. R., and Anthony, J. L. (2000). Development of emergent literacy and early reading skills in preschool children: evidence from a latentvariable longitudinal study. Dev. Psychol. 36, 596-613. doi: 10.1037//0012-1649. 36.5.596

McClelland, J. L., and Rumelhart, D. E. (1981). An interactive activation model of context effects in letter perception: part 1. An account of basic findings. Psychol. Rev. 88, 375-407. doi: 10.1037//0033-295x.88.5.375

Naka, M. (1998). Repeated writing facilitates children's memory for pseudocharacters and foreign letters. Mem. Cogn. 26, 804-809. doi: 10.3758/bf03211399

Namy, L. L., and Gentner, D. (2002). Making a silk purse out of two sow's ears: young children's use of comparison in category learning. J. Exp. Psychol. Gen. 131, 5-15. doi: 10.1037/0096-3445.131.1.5

Näslund, J. C., and Schneider, W. (1996). Kindergarten letter knowledge, phonological skills, and memory processes: relative effects on early literacy. J. Exp. Child Psychol. 62, 30-59. doi: 10.1006/jecp.1996.0021

Nelson, R. O., and Peoples, A. (1975). A stimulus-response analysis of letter reversals. J. Read. Behav. 7, 329-340. doi: 10.1080/1086296750954 7152

O'Connor, R. E., and Jenkins, J. R. (1999). Prediction of reading disabilities in kindergarten and first grade. Sci. Stud. Read. 3, 159-197. doi: 10.1207/ s1532799xssr0302_4

Palmis, S., Danna, J., Velay, J.-L., and Longcamp, M. (2017). Motor control of handwriting in the developing brain: a review. Cogn. Neuropsychol. 34, 187-204. doi: 10.1080/02643294.2017.1367654

Perry, C., Ziegler, J. C., and Zorzi, M. (2007). Nested incremental modeling in the development of computational theories: the CDP+ model of reading aloud. Psychol. Rev. 114, 273-315. doi: 10.1037/0033-295X.114.2.273

Perry, L. K., Samuelson, L. K., Malloy, L. M., and Schiffer, R. N. (2010). Learn locally, think globally: exemplar variability supports higher-order generalization and word learning. Psychol. Sci. 21, 1894-1902. doi: 10.1177/ 0956797610389189

Posner, M. I., and Keele, S. W. (1968). On the genesis of abstract ideas. J. Exp. Psychol. 77, 353-363. doi: 10.1037/h0025953

Scanlon, D. M., and Vellutino, F. R. (1996). Prerequisite skills, early instruction, and success in rst-grade reading: selected results from a longitudinal study. Ment. Retard. Dev. Disabil. Res. Rev. 2, 54-63.
Seyll, L., and Content, A. (2020). The impact of graphomotor demands on letter-like shapes recognition: a comparison between hampered and normal handwriting. Hum. Mov. Sci. 72:102662. doi: 10.1016/j.humov.2020.102662

Seyll, L., Salamin, A.-L., and Content, A. (2021). Attentional engagement in letter-like shape learning. J. Exp. Psychol. Gen. 35, 423-445.

Seyll, L., Wyckmans, F., and Content, A. (2020). The impact of graphic motor programs and detailed visual analysis on letter-like shape recognition. Cognition 205:104443. doi: 10.1016/j.cognition.2020.104443

Suggate, S., Pufke, E., and Stoeger, H. (2018). Do fine motor skills contribute to early Reading development?: fine motor skills and early reading. J. Res. Read. 41, 1-19. doi: 10.1111/1467-9817.12081

Suggate, S., Pufke, E., and Stoeger, H. (2019). Children's fine motor skills in kindergarten predict reading in grade 1. Early Childhood Res. Q. 47, 248-258. doi: 10.1016/j.ecresq.2018.12.015

Sullivan, J. V. (2018). Learning and embodied cognition: a review and proposal. Psychol. Learn. Teach. 17, 128-143. doi: 10.1177/1475725717752550

Twomey, K. E., Ranson, S. L., and Horst, J. S. (2014). That's more like it: multiple exemplars facilitate word learning. Infant Child Dev. 23, 105-122. doi: 10.1002/ icd. 1824

van Galen, G. P. (1991). Handwriting: issues for a psychomotor theory. Hum. Mov. Sci. 10, 165-191. doi: 10.1016/0167-9457(91)90003-G

Wechsler, D., and Hagin, R. A. (1964). The problem of axial rotation in reading disability. Percept. Mot. Skills 19, 319-326. doi: 10.2466/pms.1964.19.1.319

Wilson, M. (2002). Six views of embodied cognition. Psychon. Bull. Rev. 9, 625-636. doi: $10.3758 / \mathrm{BF} 03196322$

Wolff, P. H., and Melngailis, I. (1996). Reversing letters and reading transformed text in dyslexia: a reassessment. Read. Writ. 8, 341-355. doi: 10.1007/ bf00395113

Wollscheid, S., Sjaastad, J., Tømte, C., and Løver, N. (2016). The effect of pen and paper or tablet computer on early writing - A pilot study. Comput. Educ. 98, 70-80. doi: 10.1016/j.compedu.2016.03.008

Wong, K., Wadee, F., Ellenblum, G., and McCloskey, M. (2018). The devil's in the g-tails: deficient letter-shape knowledge and awareness despite massive visual experience. J. Exp. Psychol. Hum. Percept. Perform. 44, 1324-1335. doi: 10.1037/xhp0000532

Conflict of Interest: The authors declare that the research was conducted in the absence of any commercial or financial relationships that could be construed as a potential conflict of interest.

Publisher's Note: All claims expressed in this article are solely those of the authors and do not necessarily represent those of their affiliated organizations, or those of the publisher, the editors and the reviewers. Any product that may be evaluated in this article, or claim that may be made by its manufacturer, is not guaranteed or endorsed by the publisher.

Copyright (c) 2022 Seyll and Content. This is an open-access article distributed under the terms of the Creative Commons Attribution License (CC BY). The use, distribution or reproduction in other forums is permitted, provided the original author(s) and the copyright owner(s) are credited and that the original publication in this journal is cited, in accordance with accepted academic practice. No use, distribution or reproduction is permitted which does not comply with these terms. 